\title{
Characterization and simulation of peristaltic micropumps
}

\author{
M. Busek, M. Nötzel, C. Polk, and F. Sonntag \\ Fraunhofer IWS Dresden, Dresden, Germany \\ Correspondence to: M. Busek (mathias.busek@iws.fraunhofer.de)
}

Received: 27 May 2013 - Revised: 18 September 2013 - Accepted: 27 September 2013 - Published: 18 October 2013

\begin{abstract}
The aim of the work is to find an analytical model of a pneumatic micropump which was integrated into a cell-culture system. This allows the estimation of peak velocities and wall-shear stress influencing the cultured cells in our multi-organ-chip (MOC) with respect to the applied pressure and the geometric properties of the pump. By adjusting those parameters, one can imitate physiological or pathological heart activity. The calculated flow within the MOC was compared to experimental results obtained via the non-invasive micro-PIV method.
\end{abstract}

\section{Introduction}

Within the last few years, a flexible lab-on-a-chip system with an integrated peristaltic micropump has been developed (Marx et al., 2012; Sonntag et al., 2010; Wagner et al., 2013; Winkelmann et al., 2013). The pump is pneumatically actuated and establishes a pulsatile fluid flow within a closed fluidic circuit of several cell-culture segments. Each segment corresponds to a certain organ (liver, skin, kidney, etc.), forming a human-on-a-chip. This so-called multi-organ-chip (MOC) can be used by the cosmetic and pharmaceutical industries and may replace animal testing within the next few years, as predicted by Baker (2011). Each organ has a specific media uptake and should be perfused with a welldefined flow rate. Therefore a mathematical model will be used to calculate the channel distribution. Furthermore, the wall-shear stress to the adherent cells should be known. In a previous work (Schimek and Busek, 2013) the chip was cultured with endothelial cells to determine the influence of different shear stress values on the cell formation. The flow velocity was measured using micro-PIV. Those experimental results enabled us to create a mathematical model of the pump specified in this work. The model is useful especially when micro-PIV measurement cannot be applied, e.g. when the chip is filled with blood or the walls of channels are fully covered with a cell layer.

\section{Material and methods}

\subsection{Device design and fabrication}

We designed and fabricated a microfluidic multi-organ-chip (MOC) device accommodating two separate microvascular circuits, each operated by a separate peristaltic on-chip micropump. Figure 1 illustrates the system at a glance. The cover plate accommodates six air pressure fittings and four inserts forming $300 \mu \mathrm{L}$ compartments, each for media exchange and later integration of organ equivalents. The MOC holder supports controlled constant tempering of the MOC at $37{ }^{\circ} \mathrm{C}$ (Fig. 1a). Peristaltic on-chip micropumps (Fig. 1b) were modified from Wu et al. (2008). Micropump software control facilitates both clockwise and anticlockwise fluid flow. The flow rate $(Q)$ can be varied by the adjustment of the pumping frequency. Each circuit (Fig. 1c) comprises a channel volume of $10 \mu \mathrm{L}$.

Standard soft lithography and replica moulding of PDMS (Sylgard 184, Dow Corning, Midland, MI, USA) have been applied for MOC fabrication. To fabricate the microsystem, the cover plate $(\mathrm{CP})$ was treated with a silicon rubber additive (WACKER ${ }^{\circledR}$ PRIMER G 790; Wacker Chemie, Munich, Germany) at $80^{\circ} \mathrm{C}$ for $20 \mathrm{~min}$. The prepared cover plate was plugged into the master mould (channel height $100 \mu \mathrm{m}$, width $500 \mu \mathrm{m})$ and PDMS $(10: 1 v / v$ ratio of PDMS to curing agent) was injected into this casting station. The setup was incubated at $80^{\circ} \mathrm{C}$ for at least $60 \mathrm{~min}$. Teflon screws were 


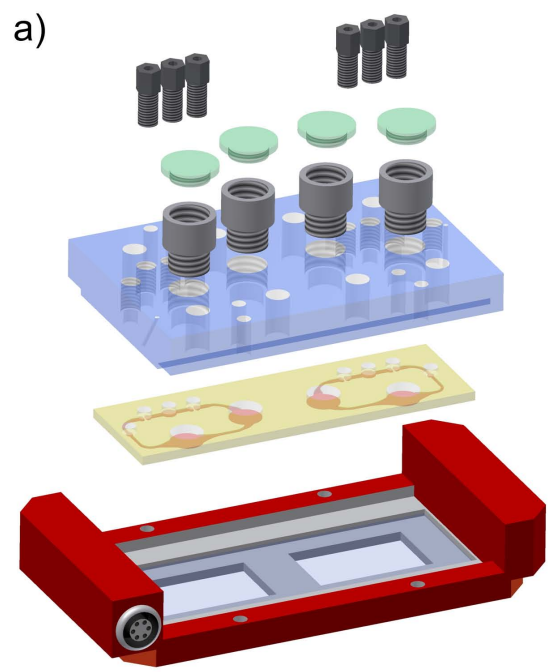

b)

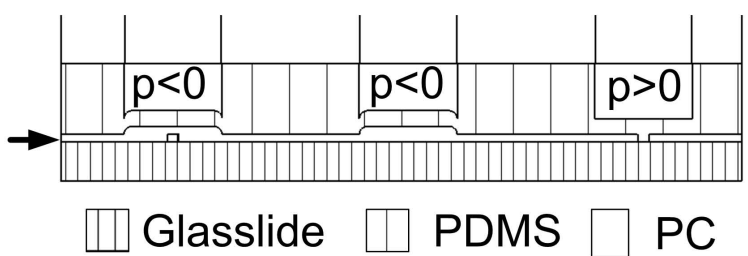

c)

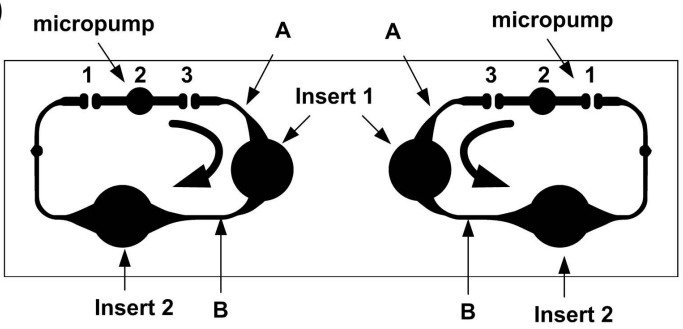

Figure 1. The microfluidic MOC device at a glance. (a) Exploded view of the device. (b) Cross-section of a peristaltic on-chip micropump; the arrow indicates the direction of flow. (c) Top view of the MOC layout illustrating the two separate microfluidic circuits. Spots A and B of each circuit designate the position of non-invasive fluid flow measurement.

used to generate the four PDMS-free culture compartments and the six $500 \mu \mathrm{m}$-thick PDMS membranes constituting the two on-chip micropumps (three membranes per micropump). The cast PDMS slice bonds fluid-tight to the CP. Thereafter, the PDMS slice attached to the CP was irreversibly bonded by low-pressure plasma oxidation treatment (Femto; Diener, Ebhausen, Germany) to a microscope slide. A sterile medium was injected immediately into the two microvascular circuits to avoid surface neutralization.

\subsection{Characterization of fluid dynamics with $\mu$ PIV}

We applied non-invasive micro-particle image velocimetry $(\mu \mathrm{PIV})$, reviewed in detail by Lindken (2009), to characterize the fluid flow in spots A and B (cf. Fig. 1c) of the microfluidic circuit. In brief, a Zeiss Primovert inverting microscope (Zeiss, Jena, Germany) with a standard halogen lamp as a continuous light source, coupled to a CMOS camera (Baumer Optronic HXC40, resolution: $2048 \times 2048$ pixels, interface: CameraLink; Baumer Optronic, Radeberg, Germany) was used to track the movement of $16 \mu \mathrm{m}$ polystyrene beads $\left(4 \times 10^{4} \mathrm{~g} \mathrm{~mL}^{-1}\right.$; Life Technologies, Darmstadt, Germany) at an exposure time of $4 \mu$ s per single image. A low magnification $(4 \times)$ was chosen to constrain the shift between two frames to approximately 50 pixels $(1$ pixel $=3.2 \mu \mathrm{m})$. The $z$ focus was set to the centre of the fluidic channel in the respective spot ( $50 \mu \mathrm{m}$ above the glass slide) to detect the peak velocity. An interrogation window at the centre of the fluidic channel $\left(1024 \times 100\right.$ pixels, $\left.3.28 \times 0.32 \mathrm{~mm}^{2}\right)$ was observed, achieving frame rates up to $3200 \mathrm{fps}$. Finally, the correlation was carried out with a software program (Fraunhofer
IWS, Dresden, Germany) which analyzes an image stack of 15000 frames, calculating the correlation maximum for the $x$ component of the displacement in a specified area. The calculated values of five successive frames are averaged to minimize artefacts. The following pump configuration was used for all experiments: pressure: $500 \mathrm{mbar}$; vacuum: $-520 \mathrm{mbar}$; and air flow: $1.5 \mathrm{~L} \mathrm{~min}^{-1}$ at $350 \mathrm{mbar}$. Figure $1 \mathrm{c}$ shows the different spots A and B where the velocity was measured.

\section{Results}

\subsection{Time-resolved micro-PIV}

We successfully applied $\mu$ PIV at the two different spots of the MOC circuits to examine the transient behaviour of the pump (Fig. 2). Figure 2 shows a full pumping cycle consisting of four steps at a frequency of $0.48 \mathrm{~Hz}$. Huang et al. $(2006,2008)$ also used $\mu$ PIV to characterize the peristaltic pump at different pumping frequencies. Their results show a good correlation with the previously obtained results (Schimek and Busek, 2013).

\subsection{Mathematical model of the pump}

The mathematical model of the pump, schematically shown in Fig. 3, is an analytical approach consisting of the following two parts:

1. Airflow from the outputs of the control unit to the deflection of the membrane

2. Liquid transport from the pumping chamber to a reservoir. 

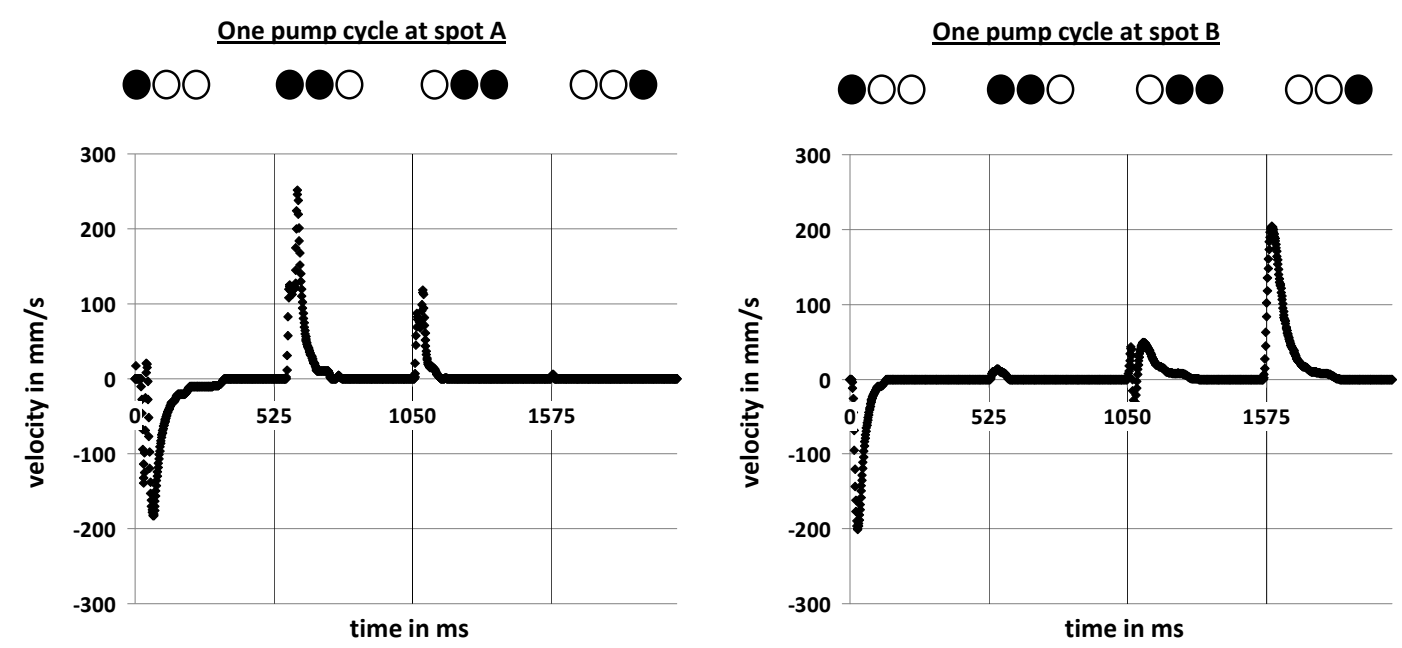

Figure 2. Exemplary velocity profiles throughout the four stages of a full pumping cycle (frequency: $0.48 \mathrm{~Hz}$ ) measured at the two discrete fluid flow analysis spots. The state of the three pump valves is represented by the circles (black = valve closed, white $=$ valve open).

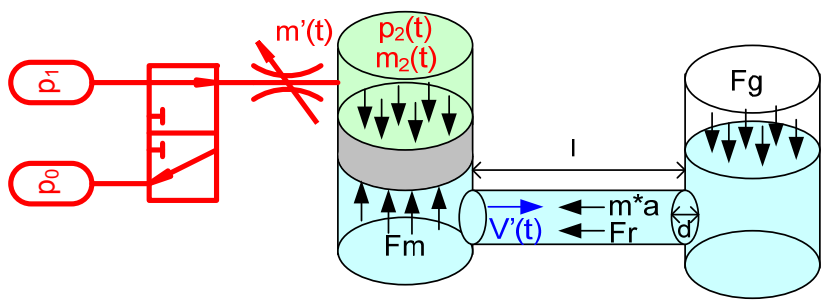

Figure 3. Schematic of the pump model with pneumatic-fluidic coupling.

By combining both parts to one differential equation, the velocity-time dependency for the liquid flowing in the channel when pressure is applied to the membrane can be calculated.

On the left side of the picture one can see the pneumatic switching circuit and the throttle which regulates the mass air flow $m^{\prime}(t)$ to the pneumatic chamber with the dead volume $V_{0}$ at the membrane. The volume $V_{0}$ is constant. Described is the moment when the valve switches from $p_{0}$ to $p_{1}$. At this moment the air inside the pneumatic chamber is compressed, which leads to a pressure and density increase. The universal gas law describes this behaviour:

$p_{2}(t)=p_{0}+R_{s} \cdot T \cdot\left(\rho(t)-\rho_{0}\right)=p_{0}+\frac{R_{s} \cdot T}{V_{0}} \cdot \int_{o}^{t} m^{\prime}(t) \mathrm{d} t$

The mass air flow through the throttle is represented by the Hagen-Poiseuille law:

$m^{\prime}(t)=\frac{\rho_{1} \cdot \pi \cdot a^{4} \cdot \Delta p}{8 \cdot \eta_{L} \cdot b}=\rho_{1} \cdot k \cdot\left(p_{1}-p_{2}(t)\right)$

The geometrical properties of the throttle ( $a \ldots$.radius, $b .$. length) as well as the dynamic viscosity of air $\eta_{L}$ are summarized as the coefficient $k$. The universal gas constant $R_{S}$, the temperature $T$ and the density $\rho_{1}$ are equal to $p_{1}$. The air flow through the throttle is therefore directly proportional to the pressure loss between both sides. The following linear differential equation describes the time-dependent pressure behaviour:

$p_{2}^{\prime}(t)=k \cdot \frac{p_{1}}{V_{0}} \cdot\left(p_{1}-p_{2}(t)\right)$

The following solution can be assumed:

$p_{2}(t)=e^{-\frac{k \cdot p_{1} \cdot t}{V_{0}}}\left[p_{0}+p_{1}\left(e^{\frac{k \cdot p_{1} \cdot t}{V_{0}}}-1\right)\right]$

Under constant pressure the membrane is deflected. The following formula describes the deflection of a round membrane with clamped edges (Timoshenko and Woinowsky-Krieger, 1959):

$z(r)=z_{\max }\left(1-\frac{r^{2}}{r_{0}^{2}}\right)^{2}$

where $z_{\max }$ describes the maximum of the elongation at the centre, $r$ is the position at the membrane and $r_{0}$ represents the membrane radius. The flow within the microchannel is equal to the displaced volume per time which can be determined by integrating this equation over the two cylindrical-space dimensions $r$ and $\phi$ :

$$
\begin{aligned}
V(t) & =\iint z(r, t) \mathrm{d} \phi \mathrm{d} r=2 \pi \cdot z_{\max }(t) \int_{0}^{r_{0}} r \cdot\left(1-\frac{r^{2}}{r_{0}^{2}}\right)^{2} \mathrm{~d} r \\
& =\frac{\pi}{3} \cdot r_{0}^{2} \cdot z_{\max }(t)
\end{aligned}
$$

$Q(t)=V^{\prime}(t)=\frac{\pi}{3} \cdot r_{0}^{2} \cdot z_{\max }^{\prime}(t)=\frac{\pi}{3} \cdot r_{0}^{2} \cdot c \cdot p_{2}^{\prime}(t)$ 


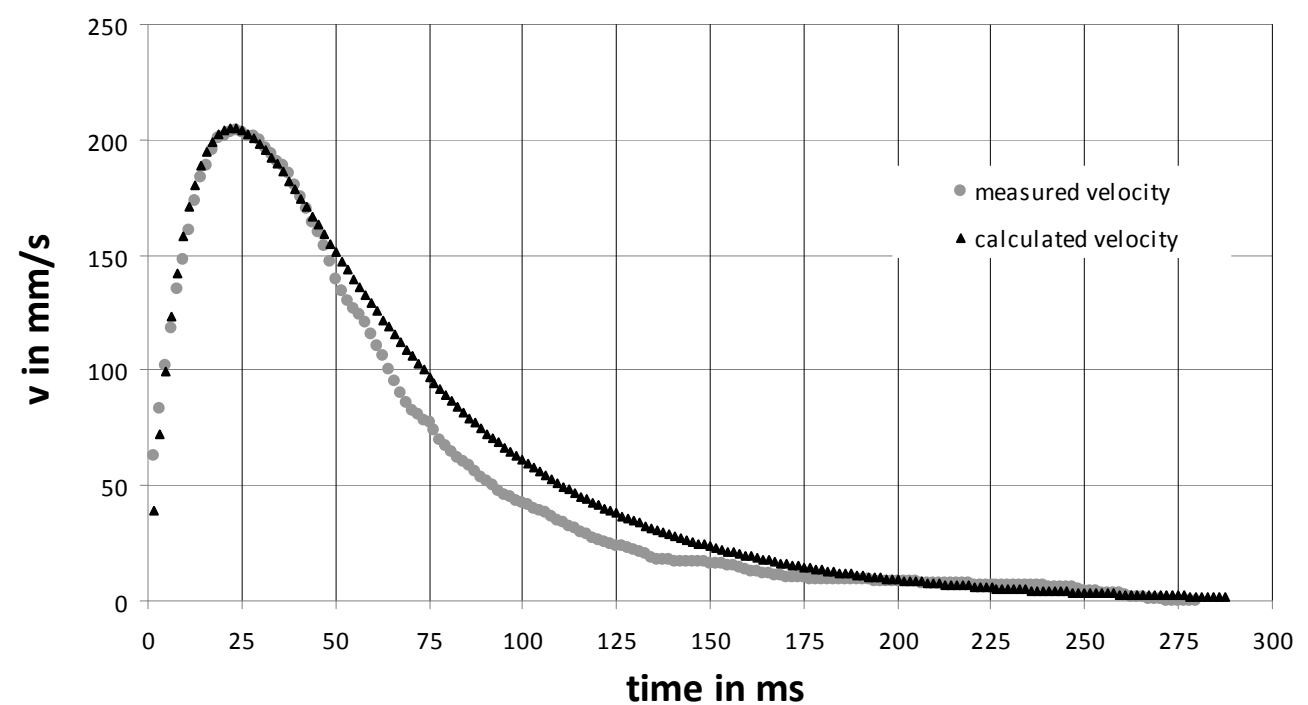

Figure 4. Calculated vs. measured velocity for one pump pulse.

As shown in Fig. 3, there are several counterforces working against the applied pressure:

1. The stiffness of the membrane, represented by the factor $c$ (Thangawng, 2007).

2. The friction force $F_{r}$ and the mass inertia $m \cdot a$ caused by the fluid flowing from the pump chamber to the reservoir through the tube-like channel.

3. The weight force $F_{g}$ counteracting by the fluid level of the reservoir.

The effective driving pressure $p_{2 \mathrm{e}}$ is represented by:

$p_{2, \mathrm{e}}(t)=p_{2}(t)-\frac{1}{r_{0}^{2} \cdot \pi}\left(F_{r}+m \cdot a+F_{g}\right)$

with the transported fluid mass $m$ and the fluid acceleration $a$. If we assume that the displaced volume is very low, then the weight force $F_{g}$ of the fluid could be neglected.

For a tube-like channel the friction force could be calculated by:

$F_{r}=8 \cdot \pi \cdot \eta \cdot l \cdot \bar{v}$

where the dynamic viscosity of the media is represented by $\eta$, the channel length is represented by $l$ and the mean velocity of the fluid in the tube is expressed by $v$.

The mass inertia can now be calculated with the fluid density $\rho_{w}$ and the channel radius $R$ :

$m \cdot a=\rho_{w} \cdot \pi \cdot R^{2} \cdot l \cdot v^{\prime}(t)$

If we differentiate Eq. (8) with respect to $t$ and insert the solution in formula (7), the volumetric flow rate can be calculated. By dividing by the channel area $\left(\pi \cdot R^{2}\right)$, one can formulate the following differential equation for the flow velocity inside the channel:

$$
\begin{aligned}
v(t) & =\frac{c}{3} \cdot\left[\frac{r_{0}^{2}}{R^{2}} \cdot \frac{k \cdot p_{1}}{V_{0}} \cdot\left\{p_{1}-e^{-\frac{k \cdot p_{1} \cdot t}{V_{0}}}\left\langle p_{1} \cdot\left(e^{\frac{k \cdot p_{1} \cdot t}{V_{0}}}-1\right)+p_{0}\right)\right\}\right. \\
& \left.-\frac{8}{r_{0}^{2}} \cdot \eta \cdot l \cdot v^{\prime}(t)-\rho_{w} \cdot l \cdot v^{\prime \prime}(t)\right]
\end{aligned}
$$

If we assume the following start and stop condition: $v(t=$ $0)=0 ; v(t \rightarrow \infty)=0$, the general solution of this differential equation is:

$v(t)=v_{\max } \cdot\left[2 \cdot e^{\left(-C_{1} \cdot t\right)}-e^{\left(-C_{2} \cdot t\right)}-e^{\left(-C_{3} \cdot t\right)}\right]$

To calculate the coefficients $C_{1}, C_{2}$ and $C_{3}$, a substitution and a numerical fitting algorithm were used. The exponents $C_{2}$ and $C_{3}$ could therefore be expressed with respect to $C_{1}$ with the three sampling points $\left(t_{0}, v_{\max }\right),\left(t_{1}=2 t_{0}, v_{1}\right)$ and $\left(t_{2}=3 t_{0}, v_{2}\right)$. The velocity-time dependency shown in Fig. 2 at spot B is used to fit the parameters of the model to the experimental data. Figure 4 shows the measured and calculated velocity for one pump pulse:

The fitted parameters for this pressure pulse are $C_{1}=19$, $C_{2}=74.5, C_{3}=97$ for $t_{0}=0.025 \mathrm{~s}, v_{\max }=204 \mathrm{~mm} \mathrm{~s}^{-1}$ and $v_{1}=121 \mathrm{~mm} \mathrm{~s}^{-1}$. This leads to the following approximation formula to calculate the pressure pulse for one pump cycle:

$v(t)=204 \frac{\mathrm{mm}}{\mathrm{s}} \cdot\left[2 \cdot e^{(-19 \cdot t)}-e^{(-74.5 \cdot t)}-e^{(-97 \cdot t)}\right]$

\section{Conclusions}

Within this work the developed peristaltic micropump was characterized with the help of non-invasive $\mu \mathrm{PIV}$ measurements and modelled using an analytical approach. In contrast to the model described by Nedelcu et al. (2007), the mass inertia and friction force of the liquid are also to be 
considered. Furthermore, the calculated results for a pump pulse of the periodic pulsatile fluid flow fit the experimental data very well. Previous findings reported by Schimek and Busek (2013) show that the described pump is suitable for the cultivation of human endothelial cells; the produced wallshear stress is within the physiological range. The developed model can be used to calculate the peak velocity with respect to the pumping parameters (pressure, diameter, etc.). In further works the influence of the elastic channel walls should be considered. It is planned to develop a network model of the MOC with a peristaltic pump based on simulation platform SimulationX, similar to the electrical equivalent network for a peristaltic pump described by Bourouina and Grandchamp (1996). This tool allows the calculation of the flow in a branched fluidic device with several cell-culture systems connected in parallel.

Acknowledgements. The authors thank the Federal Ministry of Economics and Technology for funding this work within the ZIM SimFluNet project.

Edited by: N.-T. Nguyen

Reviewed by: two anonymous referees

\section{References}

Baker, M.: A living system on a chip, Nature, 471, 661-665, 2011.

Bourouina, T. and Grandchamp, J.-P.: Modeling micropumps with electrical equivalent networks, J. Micromech. Microeng., 6, 398404, 1996.

Huang, C.-W., Huang, S.-W., and Lee, G.-B.: Pneumatic micropumps with serially connected actuation chambers, J. Micromech. Microeng., 16, 2265-2272, 2006.

Huang, S.-B., Wu, M.-H., Cui, Z., Cui, Z., and Lee, G.B.: A membrane-based serpentine-shape pneumatic micropump with pumping performance modulated by fluidic resistance, J. Micromech. Microeng., 18, 045008, doi:10.1088/09601317/18/4/045008, 2008.

Lindken, R.: Micro-particle image velocimetry ( $\mu$ PIV): Recent developments, applications, and guidelines, Lab Chip, 9, 25512567, 2009.
Marx, U., Walles, H., Hoffmann, S., Lindner, G., Horland, R., Sonntag, F., Klotzbach, U., Sakharov, D., Tonevitsky, A., and Lauster, R.: "Human-on-a-chip" Developments: A Translational Cutting-edge Alternative to Systemic Safety Assessment and Efficiency Evaluation of Substances in Laboratory Animals and Man?, ATLA, 40, 235-257, 2012.

Nedelcu, O. T., Morelle, J.-L., Tibeica, C., Voccia, S., Codreanu, I., and Dahms, S.: Modelling and simulation of a pneumatically actuated micropump, Semiconductor Conference, 2007.

Schimek, K., Busek, M., Brincker, S., Groth, B., Hoffmann, S., Lauster, R., Lindner, G., Lorenz, A., Menzel, U., Sonntag, F., Walles, H., Marx, U., and Horland, R.: Integrating biological vasculature into a multi-organ-chip microsystem, Lab Chip, 13, 3588-3598, 2013.

Sonntag, F., Schilling, N., Mader, K., Gruchow, M., Klotzbach, U., Lindner, G., Horland, R., Wagner, I., Lauster, R., Howitz, S., Hoffmann, S., and Marx, U.: Design and prototyping of a chipbased multi-micro-organoid culture system for substance testing, predictive to human (substance) exposure, J. Biotechnol., 148, 70-75, 2010.

Thangawng, A. L., Ruoff, R. S., Swartz, M. A., and Glucksberg, M. R.: An ultra-thin PDMS membrane as a bio/micro-nano interface: fabrication and characterization, Bomed. M., 9, 587-595, 2007.

Timoshenko, S. and Woinowsky-Krieger, S.: Theory of plates and shells, 2nd Edn., McGraw-Hill Book Company, 1959.

Wagner, I., Materne, E.-M., Brincker, S., Süßbier, U., Frädrich, C., Busek, M., Sonntag, F., Sakharov, D., Trushkin, E., Tonevitsky, A., Lauster, R., and Marx, U.: A dynamic multi-organ-chip for long-term cultivation and substance testing proven by $3 \mathrm{D}$ human liver and skin tissue co-culture, Lab Chip, 13, 3538-3547, 2013.

Winkelmann, C., Luo, Y., Lode, A., Gelinsky, M., Marx, U., Busek, M., Schmieder, F., and Sonntag, F.: Charakterisierung von in Lab-on-a-Chip Systemen eingebetteten Hohlfasern, Tech. Mess., 80, 147-154, 2013.

Wu, M.-H., Huang, S.-B., Cui, Z., Cui, Z., and Lee, G.-B.: A high throughput perfusion-based microbioreactor platform integrated with pneumatic micropumps for three-dimensional cell culture, Biomed. M., 10, 309-319, 2008. 